\title{
Shock wave effects in biomechanics
}

\section{B STURTEVANT}

Graduate Aeronautical Laboratories, California Institute of Technology, Pasadena, CA 91001, USA

e-mail: brad@galcit.caltech.edu

\begin{abstract}
Focused shock waves convey energy from a source and deposit it in a localized region remote from the source. This mechanism is used to deliver crushing impulses to kidney stones in patients, but it may also be an undesirable source of tissue injury. Tissue injury occurs in extracorporeal shock wave lithotripsy (ESWL), a popular procedure used around the world to treat kidney stone disease. Shock waves can also be responsible for unexpected symptoms appearing remote from the site of blunt impact trauma. This paper describes two examples of the effects of focused shock waves on tissue. A victim of gunshot wound suffered specific neurological symptoms indicating highly localized injury to the spinal cord remote from the impact point and penetration of the missile. We suggest that wave focusing by thoracic vertebrae can concentrate energy into the spinal canal, with consequent injury to the spinal cord. Impulsive stress in repeated shock waves of order $20 \mathrm{MPa}$ strength administered in ESWL is the mechanical stimulus of injury to the kidney. Research to increase the understanding of the causal relationship between the shock-induced mechanical stimulus and the consequent biomedical symptoms in the shock-wave treatment of kidney stone disease is described. The interaction of focusing shock waves with simple, planar polymeric membranes immersed in tissue-mimicking fluids has been studied. We have explored the nature of membrane failure in both cavitating and non-cavitating fluids. In water, thin nitrocellulose membranes are easily damaged during the passage of a lithotriptor shock wave by the collapse of proximal bubbles generated by cavitation. In uniform non-cavitating liquids, focusing shock waves do not by themselves cause damage, but after passing through tissue or simulated tissue, they do. Shocks with large amplitude and short-rise time (for example, in uniform media) cause no damage in non-cavitating fluids, while long-rise time, dispersed shock waves, though only moderately attenuated, do. A simple model of shearing at small scale provides a framework for accounting for the properties of the scattering medium and the membrane material in dynamic fatigue. The fact that the membranes exhibit fatigue-like behaviour suggests that a definition of dose can be formulated based on Miner's Law. This definition gives the dose at failure in terms of the stress applied to the membrane by each shock wave and the failure stress. This
\end{abstract}


analysis suggests that ESWL shock waves induce strain rates in tissue of order $10000 \mathrm{~s}^{-1}$ and that behind a tissue, phantom membranes fail after a dose of about 1400 effective shock waves, of the same order as the number of shock waves typically used in lithotripsy therapy and research. Above strain rates of about $40000 \mathrm{~s}^{-1}$, ESWL shocks cause virtually no damage.

Keywords. Focused shock waves; kidney stone disease; blunt impact trauma; extracorporeal shock wave lithotripsy.

\section{Introduction}

In this paper we describe two examples of interactions between flows and structures arising in biomechanics. In these examples the flow is induced by waves, and the structures are weak, namely, tissue. The waves are weak shocks $\left(M_{s}<1.1\right)$; nonlinear steepening and associated effects are important only near foci. The significance of the fact that in these problems waves generate motion and stress in the material is that waves can carry energy for large distances, so the effects of an action can occur at remote locations. This fact is exploited in the shock wave treatment of kidney stone disease, called extracorporeal shock wave lithotripsy (ESWL). In addition, tissue is acoustically inhomogeneous, so it refracts and scatters waves, and the resulting material motions are spatially and temporally variable. When the wave fronts become curved, as in ESWL, they can focus and the wave energy can be substantially concentrated in regions far from the source. In ESWL focused shocks producing compressive stress of order 500 bar are used to crush kidney stones. Specialists in mechanics might naturally suspect that stress sufficient to break stones should also cause damage to soft tissue. However, this fact was not appreciated during the early applications of ESWL. In fact, the effect on the human body of ESWL is similar to that of blunt impact trauma (Kaude et al 1985), so ESWL is in a class of processes which cause injury. Though injury by blunt trauma has been of concern for hundreds of years, the various mechanisms by which it can initiate and progress are still not fully understood. Yet, there are presently an increasing number of proposals to use shock-wave therapy in the treatment of a variety of diseases, including cancer, the occurrence of other accretions such as gall stones and salivary stones, and even cerebral embolism. Thus it is ever more important that the mechanisms of injury be better understood. In the next sections we review two examples in which (a) we hypothesize a possible mechanism of remote injury by gunshot, and (b) a multidisciplinary study of injury in ESWL is beginning to yield results.

\section{Remote spinal injury caused by missile penetration}

The study of 'wound ballistics' has succeeded in categorizing the mechanisms of injury from gunshot into four distinct processes (Janzon et al 1988): (i) direct missile contact with tissue, causing disruption of the tissue; (ii) high over-pressure in the immediate vicinity of the projectile, which crushes the tissue; (iii) expansion of the temporary cavity after passage of the projectile, stretching and tearing tissue, and (iv) Rayleigh-Taylor instability of the 
temporary cavity upon collapse, tearing and disrupting the cavity wall. The expansion of the temporary cavity is the consequence of the high over-pressure and vice versa. Furthermore, the pressure propagates away from the projectile as a wave at approximately the speed of sound in the tissue, and, depending on the nature of the intervening tissue, may displace and compress tissue remote from the bullet trajectory. Laboratory experiments with animals (Ming et al 1988; Suneson et al 1988, 1990) have demonstrated that gunshot injury can occur in tissue remote from the trajectory of the missile. The Brown-Séquard syndrome, a specific set of neurological symptoms resulting from spinal cord injury (Brown-Séquard et al 1860), has been reported as a consequence of gunshot wound (Taylor \& Gleave 1957; Saxon et al 1982).

\subsection{Case report}

On 8 February 1993, a 19-year old woman was shot in the left side of the posterior thorax by a 36-caliber bullet which passed through an interior wall of a residential structure in Los Angeles, struck the victim, and penetrated from the entry wound to the left lung through $14 \mathrm{~cm}$ of fatty tissue. X-ray images (figure 1) showed the bullet with multiple fragments along the 8th posterior left rib. The top of the bullet was $5 \mathrm{~cm}$ from the centre of the spinal canal. We infer that the path of the bullet from entry to stopping point was as shown in figure 2. A computed tomogram with intrathecal contrast media (figure 3 ), showed mild enlargement of the spinal cord from $\mathrm{T}_{8}$ through $\mathrm{T}_{10}$. Üpon neurological examination the patient showed symptoms typical of Brown-Séquard syndrome.

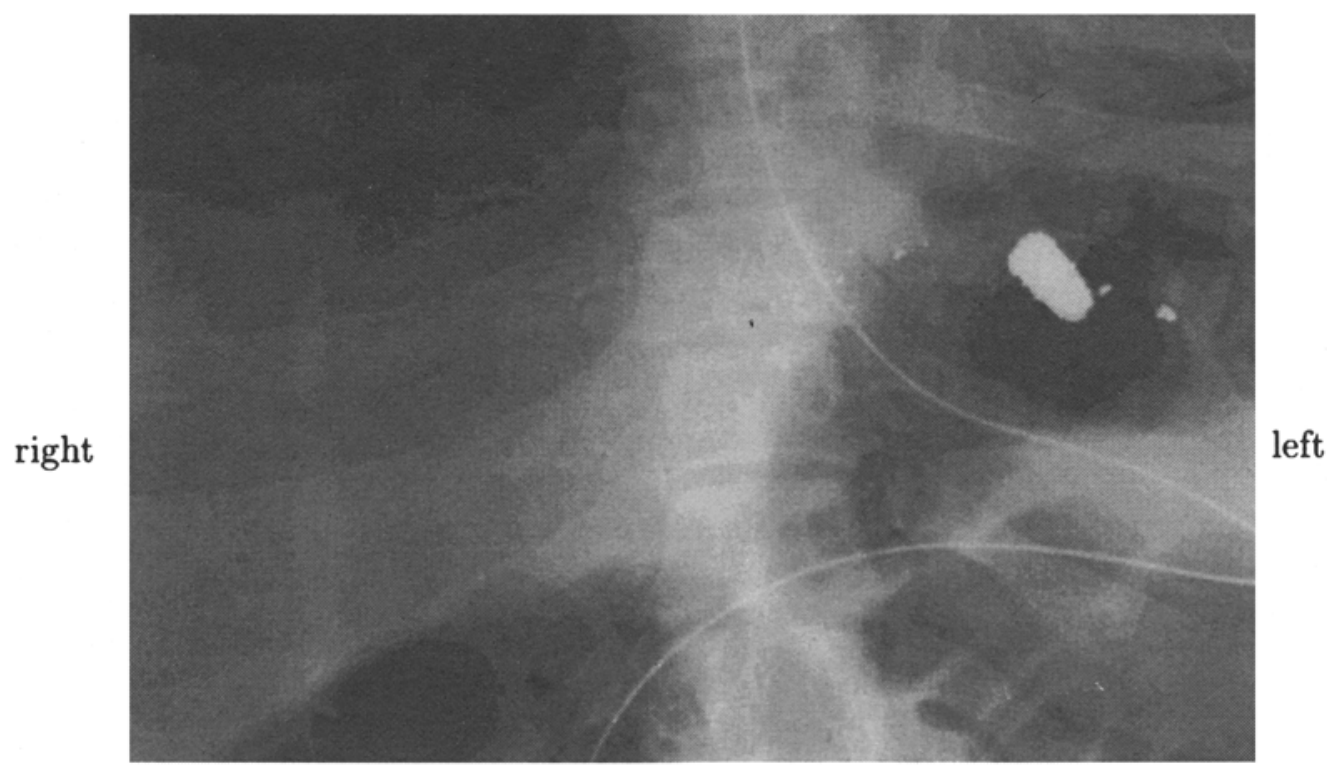

Figure 1. Axial view of the thoracic spine showing a bullet and multiple fragments along the 8 th left posterior rib. Some metal fragments are seen overlying the posterior 8 th vertebral body. With presumed $20 \%$ magnification the top of the bullet is $5 \mathrm{~cm}$ from the centre of the spinal canal, 18-2-1993. 


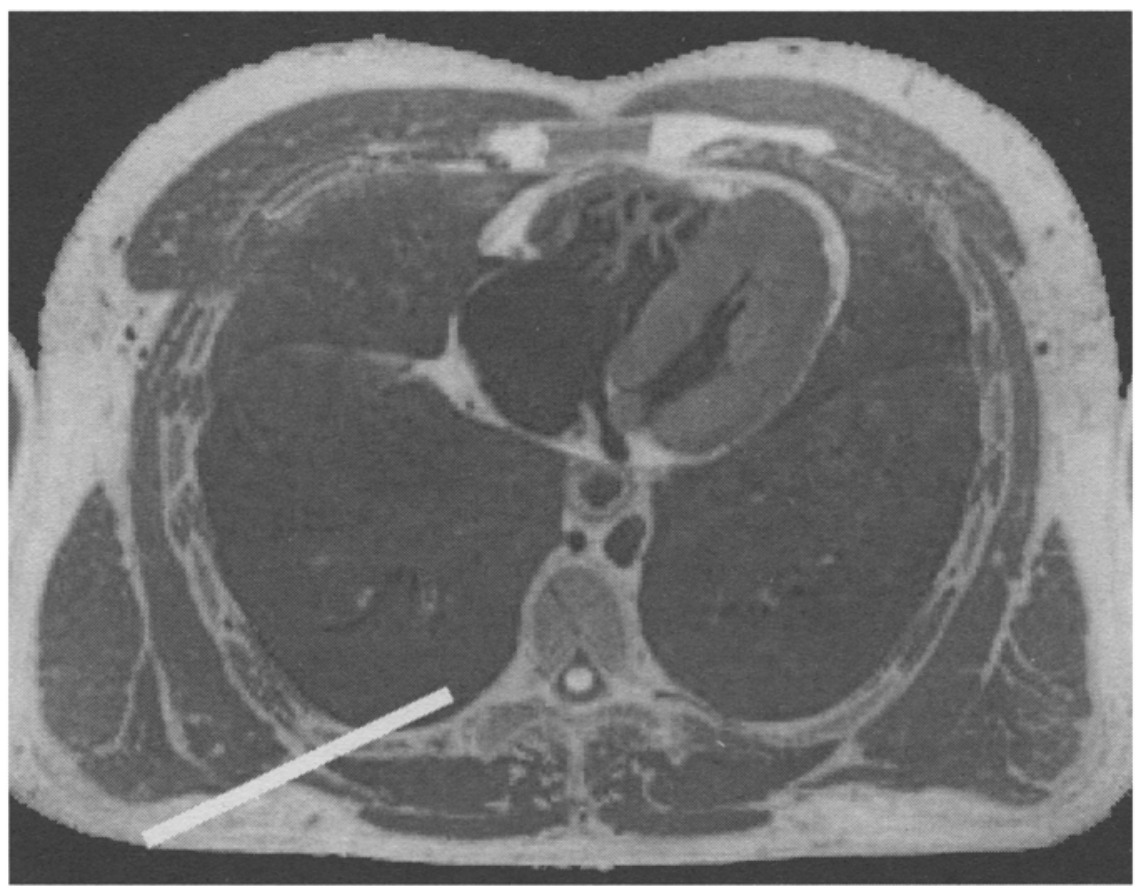

Figure 2. Presumed trajectory of the bullet from entry to point of arrest, indicated on a model transverse crossection at $\mathrm{T}_{8}$ (model courtesy of $\mathrm{J}$ A McNulty, Loyola University, Chicago).

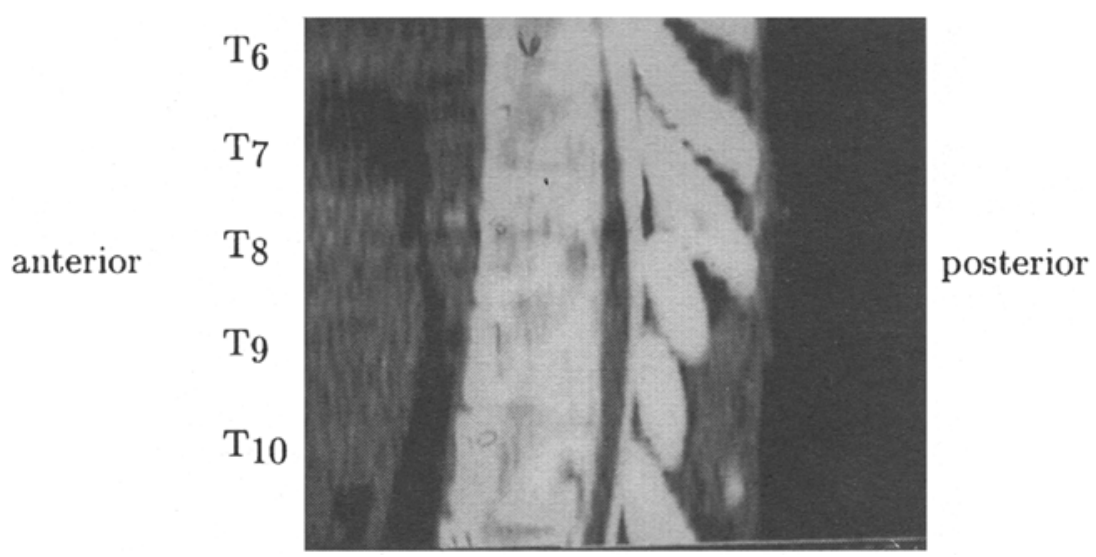

Figure 3. CT scan of the thoracic spine with intrathecal contrast. The spinal cord is intact. is normal in size at $T_{6}$, but shows mild enlargement from $T_{8}$ through $T_{10}$. The spinal cord returns to normal size at $T_{11}$. No metal fragments or fracture are seen within either the spinal cord or the neural foramina. 19-2-1993. 


\subsection{Analysis}

The focusing effect of thoracic vertebrae on pressure waves generated by missiles approaching the spine from different directions can be calculated using the theory of geometrical acoustics. The calculations were performed by the method of ray tracing. We consider three media, (1) the tissue surrounding the vertebra, (2) the bony vertebra and (3) the spinal fluid and cord within the vertebral column. These three media are separated by the two surfaces comprising the vertebra. The media are assumed to be uniform except at the boundaries, so the rays are straight between surfaces and refract at each surface according to Snell's law. An outline of the superior aspect of a typical thoracic vertebra was digitized from a figure in a standard anatomy reference (Williams \& Warwick 1989).

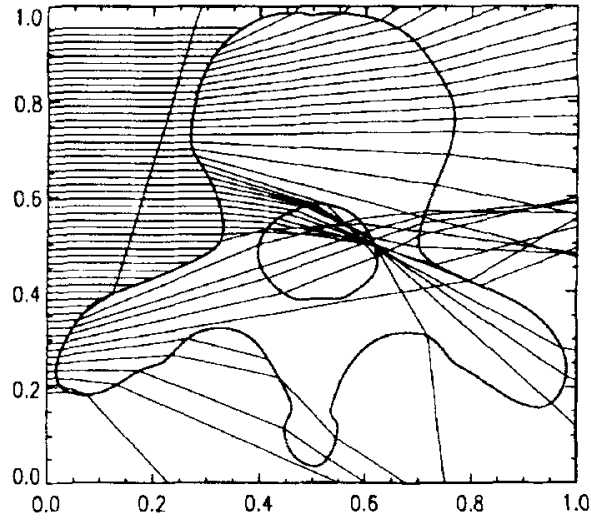

(a)

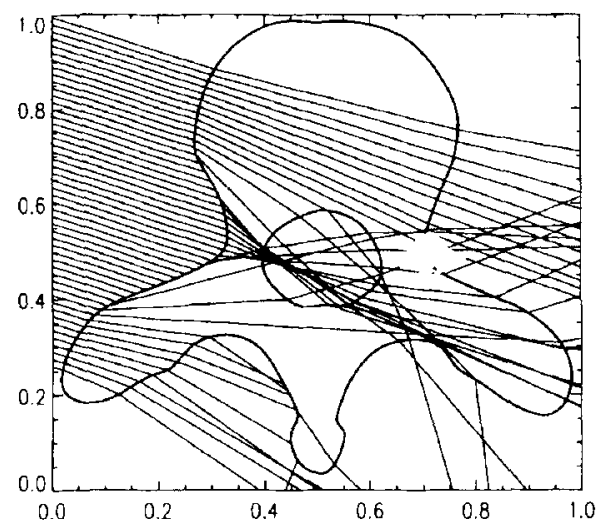

(c)

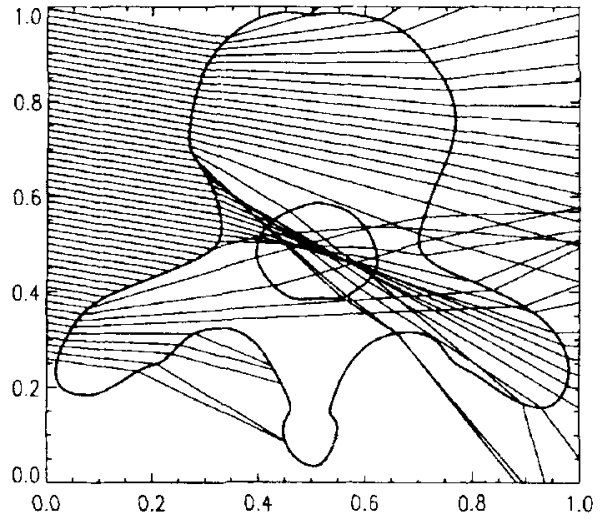

(b)

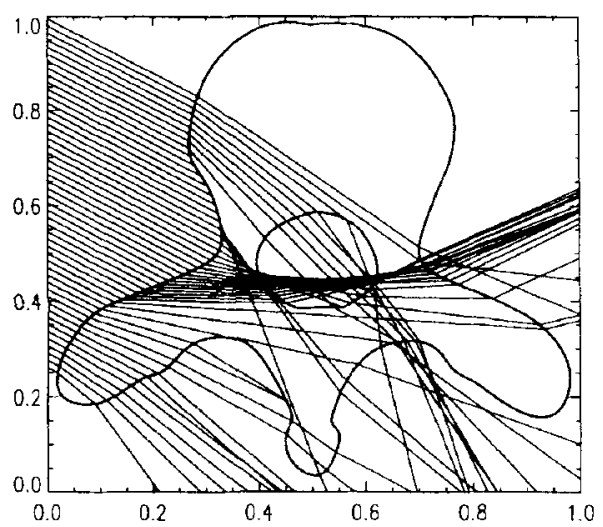

(d)

Figure 4. Focusing effect of vertebral body on a plane wave (parallel rays) incident medially from the left at four angles, illustrated by ray tracing. Incident and transmitted rays at each interface are shown; reflected rays are not shown. Sound velocity in exterior tissue, $1470 \mathrm{~m} / \mathrm{s}$; in vertebra, $2240 \mathrm{~m} / \mathrm{s}$; in spinal canal, $1750 \mathrm{~m} / \mathrm{s}$. Angle of incidence: (a) $0^{\circ}$; (b) $10^{\circ}$; (c) $20^{\circ}$; (d) $30^{\circ}$. 
For simplicity we consider the interaction of a plane wave incident medially on a vertebra represented only in a two-dimensional transverse plane. A plane wave represents the limiting case of a very distant missile. Depending on the actual location of the missile trajectory the fact that the incident wave may be diverging could change the results in detail, but the plane wave illustrates the important qualitative effects. In approximating the vertebra as a two-dimensional object we ignore the additional focusing effect of the three-dimensional concave waisted profile of the vertebral body seen in anterior aspect.

The sound velocity of the exterior tissue is taken to be that of typical fatty tissue (Goss et al 1978). The sound velocity of cancellous bone which makes up most of the vertebral body has not been reported, so a value less than that reported for cortical bone and one typical of the skull (Goss et al 1978) has been used. Similarly, the sound speed of tissues of the spinal canal is not available, so a value typical of tendinous tissues (Goss et al 1978) has been adopted. Figure 4 shows the ray configurations for four different incidence angles. It can be seen that, owing to the large sound velocity in bony tissue, the concave shape of the vertebral body and pedicle can act as a focusing lens over a large range of incidence angles. Focusing is indicated by the occurrence of caustics.

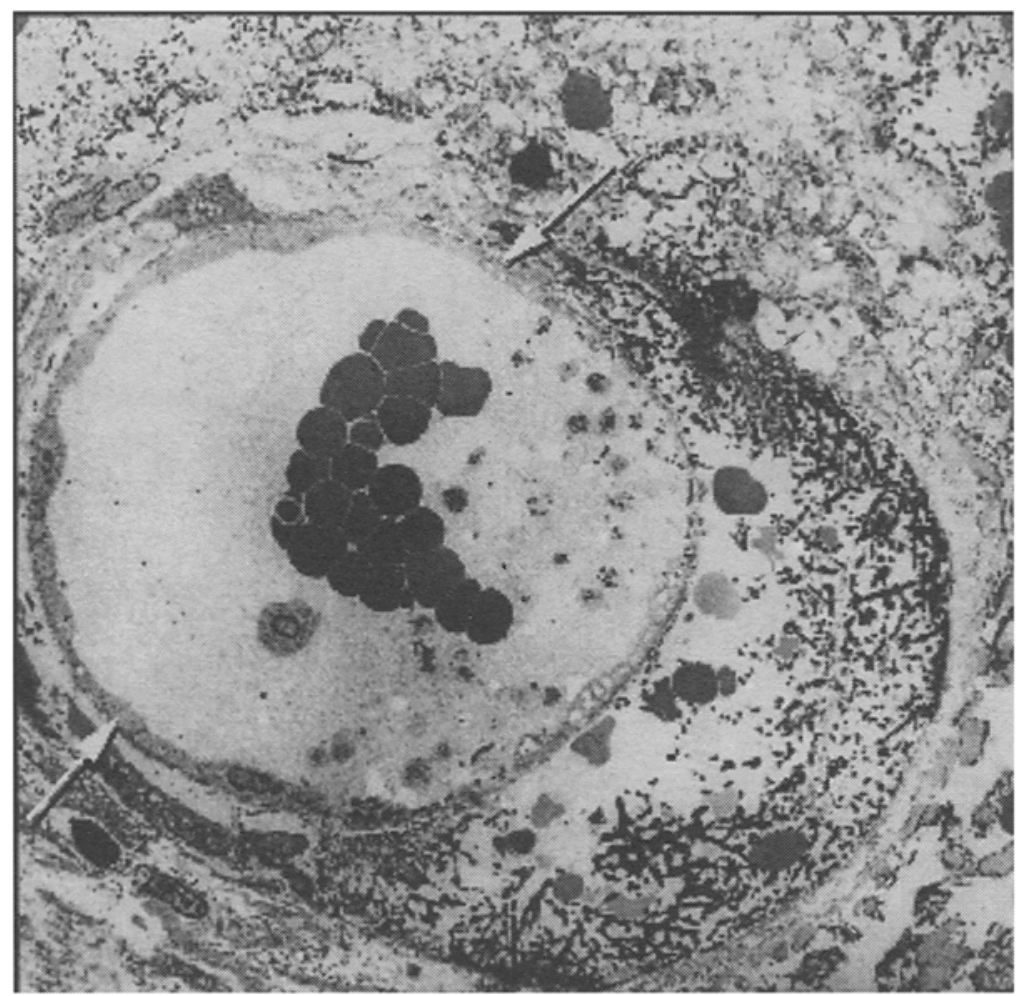

Figure 5. Cross-section of a damaged artery in which the enlarged tunica media entirely lacks smooth muscle cells (to the right of arrows), suggesting that they have been liquefied, and contains numerous red and white blood cells and cellular debris. Note the damaged endothelial cells in that region compared to the uninjured portion. 


\section{Mechanisms of injury by ESWL}

\subsection{Progression of injury in an animal model}

Experiments on a pig model are being carried out by colleagues at Indiana University School of Medicine, Indianapolis, to investigate the progression of injury by ESWL at the systemic level (Evan \& McAteer 1996; Evan et al 1996). In this section we briefly report some of their results. They have found that injury first occurs at the micro-vascular level. Shock waves lacerate and tear the walls of veins and capillaries at the focal point of the shock wave (known as "F2"), producing a unique injury by removing endothelial cells. In their place, platelets and polymorphonuclear cells attach. Arteries also exhibit a unique effect; a swelling of the smooth-muscle region of the vessel wall (tunica media). This causes extensive narrowing of the vessel lumen. Rupture of the artery occurs in the region where the tunica media has swelled. This occurs via a complete breakdown of the endothelial sheath which normally is in contact with the blood, allowing hemorrhage of blood out of the vessel. Figure 5 shows an example where one half of an artery is normal (left) and the other is severely injured (right). The microscopic lesions grow with additional shock waves, merge and eventually, after one or two thousand shocks, pervade the focal region. The result at the macro level is severe lesioning of the kidney tissue (figure 6).

The important consequence from the point of view of the patient's well-being is the volume of functioning kidney tissue, which performs the crucial filtering function of the kidney, permanently lost during the shock wave procedure. Methods have been developed at Indianapolis to quantify the volume of the kidney which is lesion after the treatment

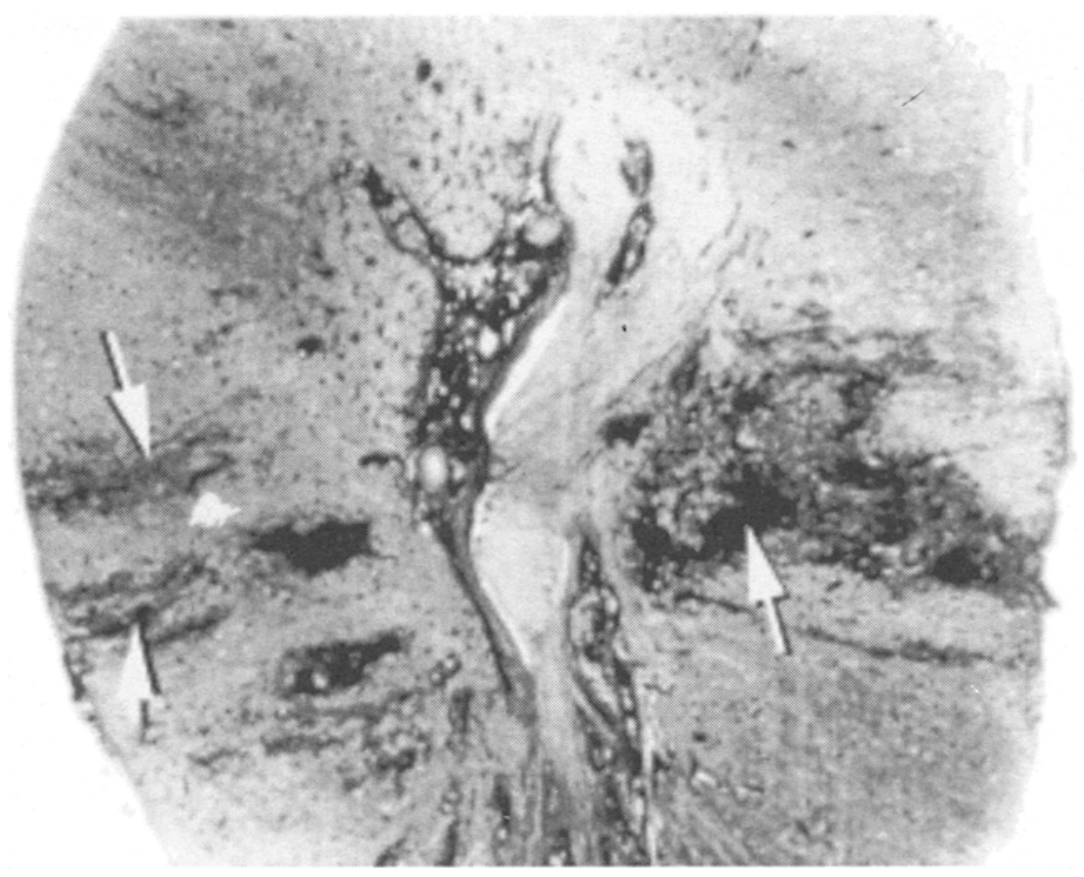

Figure 6. Acute lesions (arrows) seen 24 hours after treatment. 

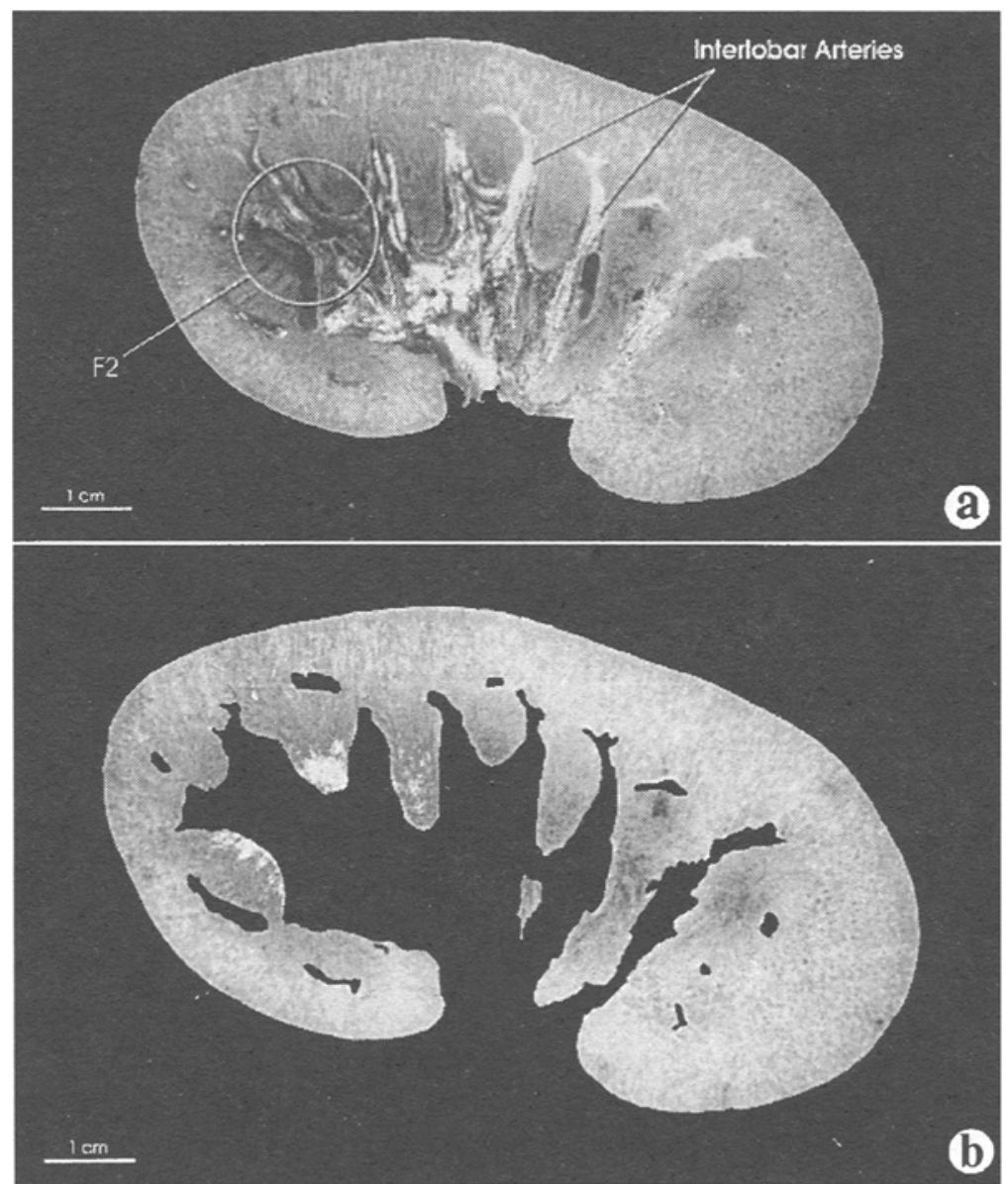

Figure 7. An example of serial subsections taken through ESWL-treated kidneys. Injured area is darkened region near F2 (a). For quantitative measurements of the injured volume, the non-parenchymal regions of the kidney are digitally removed, and the lesions highlighted (b).

(figure 7). Movies were made of these serial subsections passing sequentially through the kidney to show the injuries in $3 \mathrm{D}$.

\subsection{In vitro study of the mechanical effects of ESWL}

Understanding of the causal relationship between the shock-induced mechanical stimulus and the consequent biomedical symptoms described above will facilitate steps to reduce the injury and improve the efficiency of ESWL. Two physical mechanisms, one direct and the other indirect, are possible explanations of injury to soft tissue by ESWL. The direct mechanism is shearing and tearing induced by shock scattering from small-scale tissue inhomogeneity. The indirect effect is pressurization caused by cavitation bubble collapse. In this paper we consider the direct, shock-scattering effects.

The focused shock wave from a lithotriptor first compresses the tissue for about $1 \mu \mathrm{s}$ and then puts it into tension for a few $\mu \mathrm{s}$. In addition, since the strength of the focused 
shock varies laterally along the wave front by scattering from microscopic cellular acoustic heterogeneity, shearing also occurs. When the shock wave trajectory is perpendicular to the axes of bundles of tubules and vesicles that are found in the kidney, it is most strongly scattered by the acoustically varying properties of blood, urine, vessel walls, epithelial tubules and collagenous interstitium. The result is that the shock becomes contorted and thickened, and focusing and defocusing occur, with consequent substantial lateral variation of strength along the front, on the scale of the vessels.

In experiments at Caltech (Howard \& Sturtevant 1997), the effects of focused shock waves on thin planar polymeric membranes immersed in a variety of tissue-mimicking fluids have been examined. The membranes and fluids used in this study are not biological, but the present mechanical approach has the advantage that membranes of large surface area can be studied, membrane position and orientation and the shock environment can be more accurately controlled, and shock wave effects can be more precisely quantified. With this arrangement, shock-wave effects on weak structural elements simulating the mechanical properties of tissue have for the first time been studied. We explore the nature of membrane failure in both cavitating and non-cavitating fluids. A direct mechanism of failure by shock compression and an indirect mechanism by bubble collapse are observed. In homogeneous media such as a uniform, non-cavitating liquid, compression by a focusing shock wave does not cause damage to membranes, but in heterogeneous media, like tissue, shock waves cause membranes to fail by a progressive shearing mechanism.

In many materials, including polymeric solids, the development of damage during dynamic fatigue depends not only on the applied stress or strain but also on the rate at which the strain is applied and the accumulated number of strain cycles. As the strain rate increases, the failure stress and the number of stress cycles to failure increase; the material becomes more resistant to failure. The more a shock wave is dispersed by propagation in a heterogeneous medium like tissue, the more the strain rate decreases, and therefore the more the tissue is subject to damage. In other words, the inhomogeneity of tissue contributes to its own injury by reducing the strain rate. However, an attendant protection is afforded by attenuation of the shock wave. Recognition of these facts is crucial to the analysis of ESWL kidney injury and to the development of methods for its amelioration. In present practice, the dose administered during ESWL is measured by the lithotriptor settings (e.g., the power level) and the number of shots. With dose defined in terms of

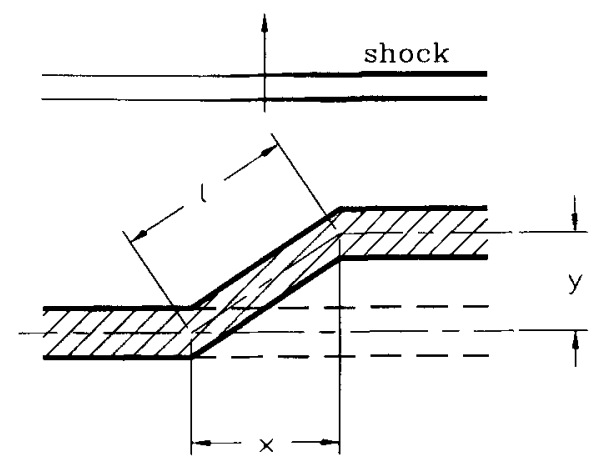

Figure 8. Sketch of the shear model. The shock wave is at the top, having passed through the membrane. The postshock membrane is indicated by the hatched area, preshock, by the dashed lines. $x$ is the lateral scale of strength variations along the shock front, assumed approximately parallel to the membrane, $y$ is the amount of differential motion that occurs during passage of the ESWL pulse. $l$ is the new length of the stretched portion of the membrane, previously $x$. 
the principles of dynamic fatigue, it is possible to derive damage criteria and thresholds in terms of the physical parameters of the lithotriptor and target systems which will accurately reffect the effects of the cumulative damage from ESWL.

A simple model of shearing on a small scale provides a framework for accounting for the properties of the scattering medium and the membrane material. Focusing and defocusing of the shock front by the variable acoustic properties of the medium cause the shock strength to vary laterally along the front on the scale of the size of the heterogeneities, so relative shearing motion of the membrane occurs, as indicated schematically in figure 8 . $x$ is the scale of the heterogeneity and $y$ is the differential motion induced by the variation of shock strength across the distance $x$, indicated by the variations in thickness of the horizontal lines at the top which signify the passing shock. We assume that the change of strength is the same order as the strength of the shock wave itself, that is, that the shock strength is roughly doubled or halved by typical focusing and defocusing action. With this assumption,

$$
y \doteq u \Delta t=\left(p_{\max }^{+} / \rho a\right) \Delta t
$$

where $u$ is the shock-induced fluid velocity and $\Delta t$, the duration of the streaming, is taken to be half the full pulse width of the lithotriptor shock field. The relationship between $u$ and $p_{\max }^{+}$used in the second equality of (1) results from acoustic theory; $a$ is the speed of sound in the medium and $\rho a$ is its acoustic impedance. In the shearing model the stress applied to the membrane between the horizontal sections in figure 8 is tensile. The stretched length

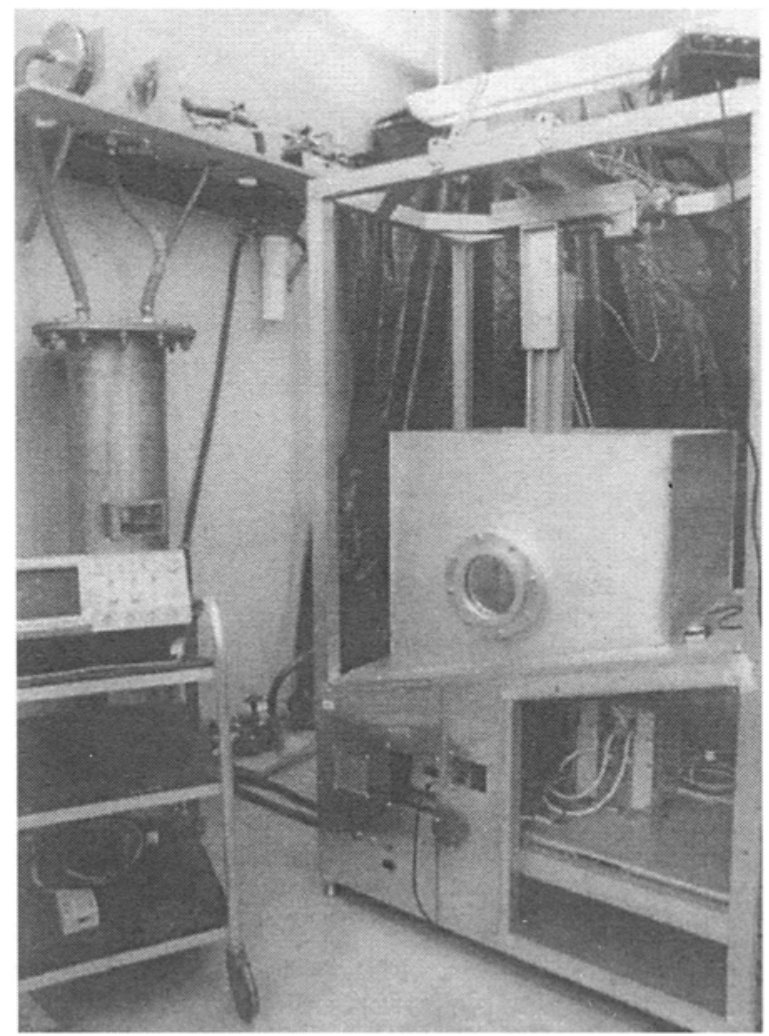

Figure 9. Experimental lithotriptor designed to simulate an unmodified HM3. Computer-controlled positioning system shown over tank with optical window. Power supply visible under tank. Water treatment system on wall at left. 
$l$ of the membrane was initially $x$, and after shock passage is

$$
l=\left(x^{2}+y^{2}\right)^{1 / 2} \text {. }
$$

Therefore, in this model the strain $\epsilon$ is

$$
\epsilon \equiv[(l-x) / x]=\left(1+(y / x)^{2}\right)^{1 / 2}-1 .
$$

For plane stress

$$
\sigma=E \epsilon,
$$

where $E=5 \times 10^{9} \mathrm{~Pa}$ is the bulk modulus of nitrocellulose. We retain the shock rise time $\tau$ as the characteristic time, rather than pulse width $\Delta t$, because $\tau$ is much more sensitive to the effects of scattering in heterogeneous media than is $\Delta t$. Thus the magnitude of the strain rate is

$$
\epsilon=\epsilon / \tau \text {. }
$$

3.2a Experimental equipment: An experimental electrohydraulic lithotriptor has been designed and built to simulate the shock-wave characteristics of a Dornier HM3 "unmodified" machine (figure 9). The layout of the experimental apparatus is shown schematically in figure 10 . Shock waves are generated by an underwater spark gap powered by a highvoltage pulsed power supply of local design. The spark plugs are positioned with the gap

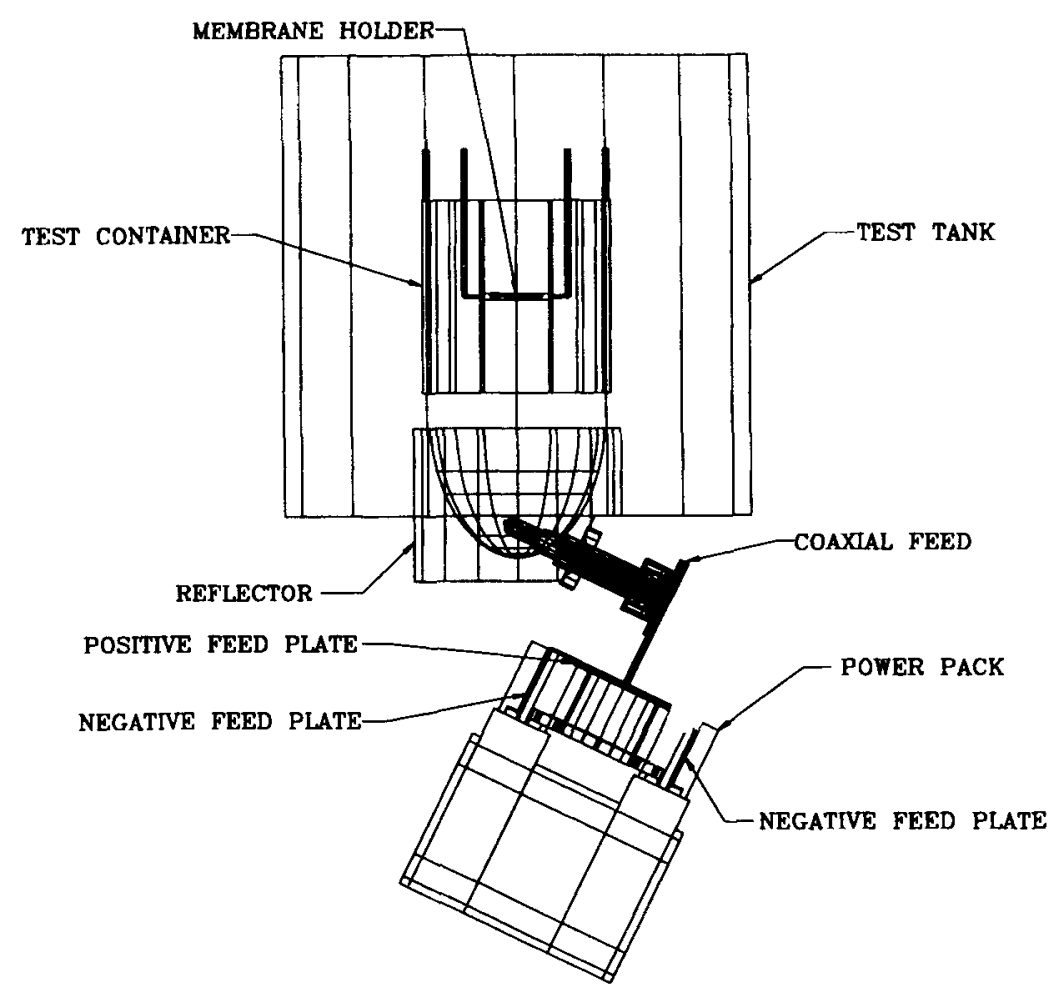

Figure 10. Schematic of the layout of the experimental lithotriptor. 
at the focus of a truncated ellipsoid of brass of semi-major axis $138 \mathrm{~mm}$ and semi-minor axis $77.5 \mathrm{~mm}$, truncated by $15 \mathrm{~mm}$. The ellipsoid is contained in an aluminum water tank whose bottom is tilted at $14.3^{\circ}$ to the horizontal, $2 \times 2.5 \times 2 / 1.5 \mathrm{ft}$ deep. The test objects are placed at F2, the second focus of the ellipse. The test liquids and membrane specimens are contained in a $16.5 \mathrm{~cm}$ (ID) by $19.0 \mathrm{~cm}$ high polyvinyl chloride (PVC) cylindrical vessel suspended in the electrolytic fluid of the lithotriptor tank. The bottom of the test container is made of an $80 \mu \mathrm{m}$ thick low-density polyethylene sheet, the acoustic impedance of which is 1.17 times that of water. The pressure transducers, test specimens etc. are placed inside the test container $7.5 \mathrm{~cm}$ above its polyethylene bottom. The test container and transducer or membrane translate together relative to the test tank and F2 on a three-axis lead-screw traverse.

Shock pressures are measured with piezoelectric transducers made from poled polyvinyli. dene fluoride (PVDF) plastic coupons $25 \mu \mathrm{m}$ thick. The minimum measured rise time (10\%-90\% peak amplitude) of a transducer sheathed in a non-cavitating liquid (castor-oil) and immersed in water at F2 is 37 ns. To provide a control on cavitation, tests were conducted in four different liquids. In the order of their tendency to cavitate, they are castor oil, glycerine, ethylene glycol, and distilled water; water cavitates extensively in a lithotripter shock field and castor oil not at all.

Membranes used to simulate weak tissue-like structures are made of nitrocellulose and are approximately $2 \mu \mathrm{m}$ thick and $4.1 \mathrm{~cm}$ in diameter. The membranes are oriented horizontally in the test container (perpendicular to the blast path). Figure 11 shows a membrane before immersion in water.

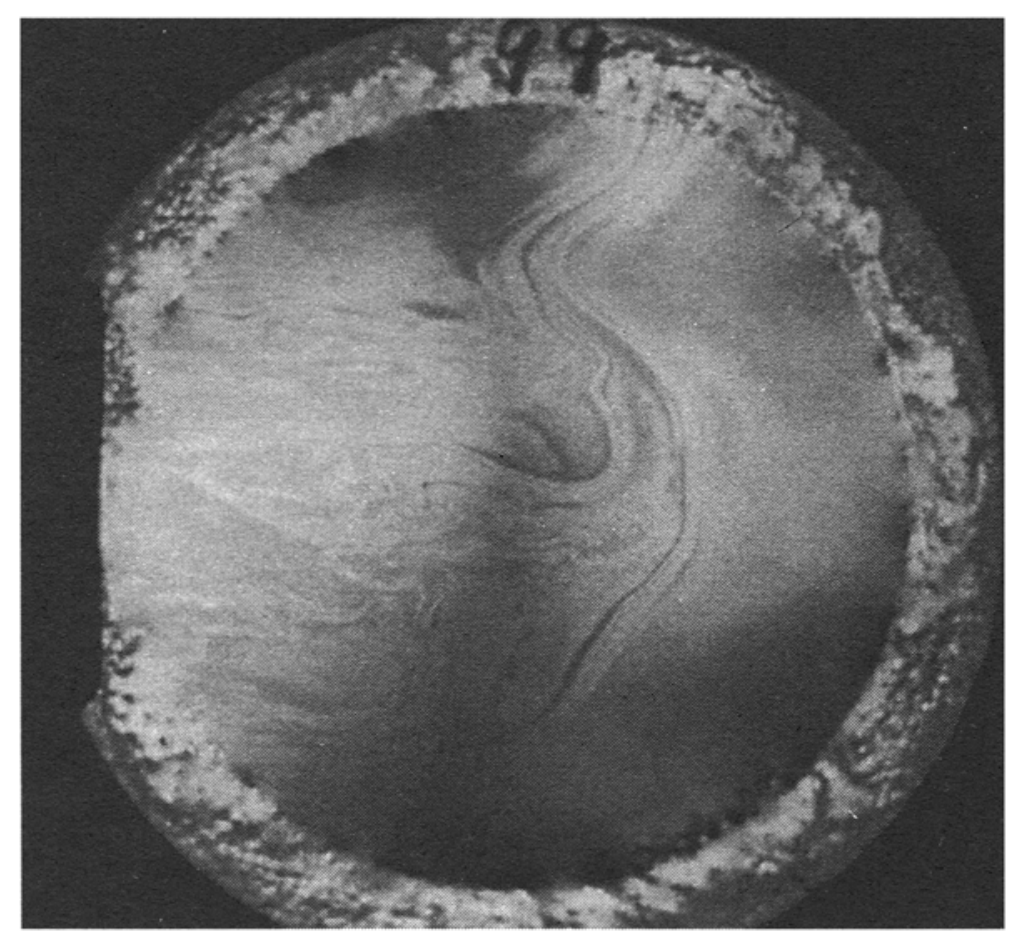

Figure 11. Nitrocellulose membrane before immersion in test liquid and application of shock waves. 


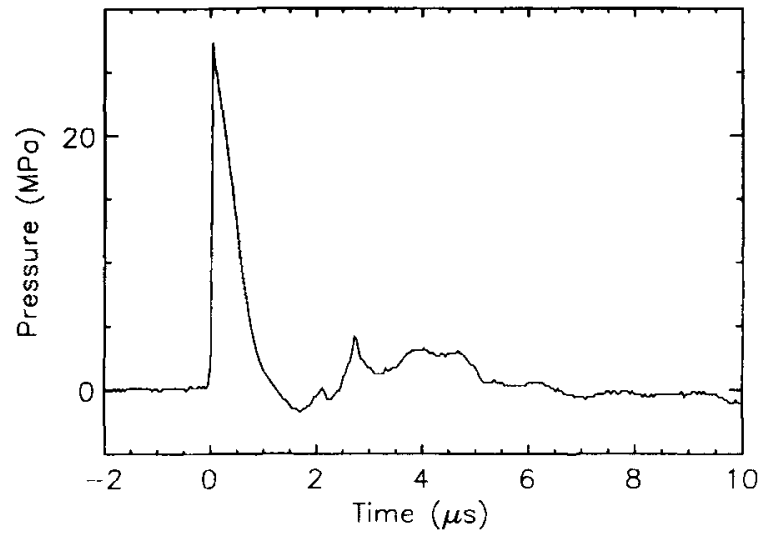

Figure 12. Typical pressure trace at $\mathrm{F} 2$ in water. Discharge voltage $18 \mathrm{kV}$.

Since focused shock waves in uniform non-cavitating liquids do not damage membranes, phantoms are used to simulate the effects of wave scattering by small-scale variations of the acoustic properties of tissue. First, to determine whether small-scale variations of shock strength caused by scattering can cause damage to membrane structures, we used strongly scattering hollow glass spheres mixed into the test liquid. The spheres are of mean diameter $65 \mu \mathrm{m}$, wall thickness approximately $1.2 \mu \mathrm{m}$ and mean density $238 \mathrm{~kg} \mathrm{~m}^{-3}$. The concentrations $\alpha$ of glass spheres that are used, and the corresponding mean separation between the spheres are as follows: $10 \mu \mathrm{I}^{-1}, 460 \mu \mathrm{m} ; 40 \mu \mathrm{I}^{-1}, 290 \mu \mathrm{m} ; 160 \mu \mathrm{I}^{-1}, 180 \mu \mathrm{m}$; $1600 \mathrm{Ll}^{-1}, 86 \mu \mathrm{m}$. Second, to test the effects of scattering in real tissue, animal tissue obtained at a supermarket is immersed in the test liquid immediately upstream of the membrane specimen at F2. In one test, a slab of lean beefsteak $3.8 \mathrm{~cm}$ thick is used and in another, pork skin, muscle, fat and kidney tissue are layered in slabs $0.23,1.9,1.0$ and $3.0 \mathrm{~cm}$ thick respectively.

Figure 12 shows a typical pressure trace at $\mathrm{F} 2$ in water. Its features are typical of waveforms generated by electrohydraulic lithotriptors; rise time $\tau=50 \mathrm{~ns}$ (defined as the time for the signal to rise from baseline to peak), pulse width $1 \mu \mathrm{s}$ and total duration of shock signal $4 \mu \mathrm{s}$. The slab of beef immersed in castor oil attenuated lithotriptor shock waves much more than in pure water or castor oil (figure 13). $\tau$ was increased to $400 \mathrm{~ns}$.

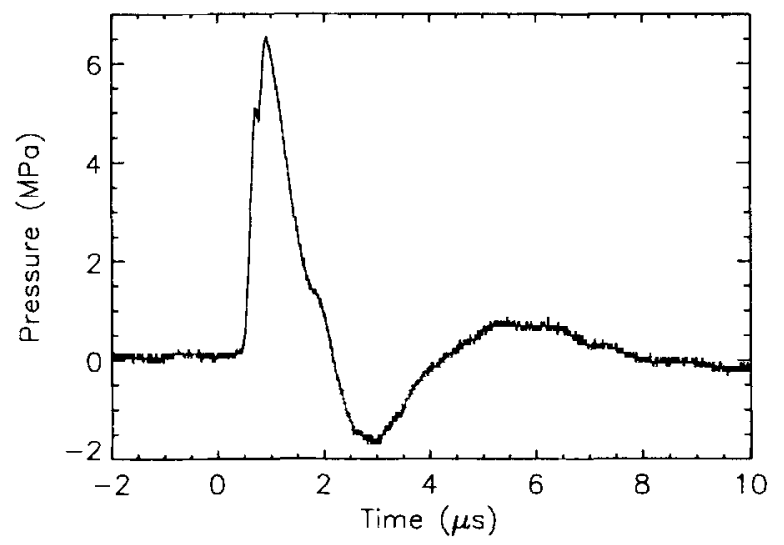

Figure 13. Pressure at the geometrical F2 behind $3.8 \mathrm{~cm}$ of muscle tissue. Note that the vertical scale is different from that in figure $12 . \tau=400 \mathrm{~ns}$. 


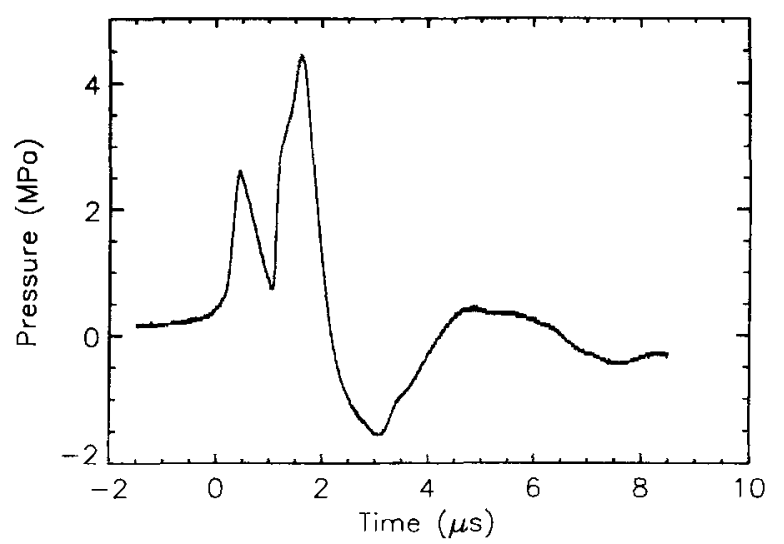

Figure 14. Pressure at the geometrical F2 behind layered tissues $6 \mathrm{~cm}$ thick. Note that the vertical scale is different from those in figures 12 and 13. $\tau=1.8 \mu \mathrm{s}$.

The layered stack of pork skin, muscle, fat and kidney caused larger attenuation, and nonparallel layers of tissue caused large refraction and the formation of multiple peaks on the waveform (figure 14).

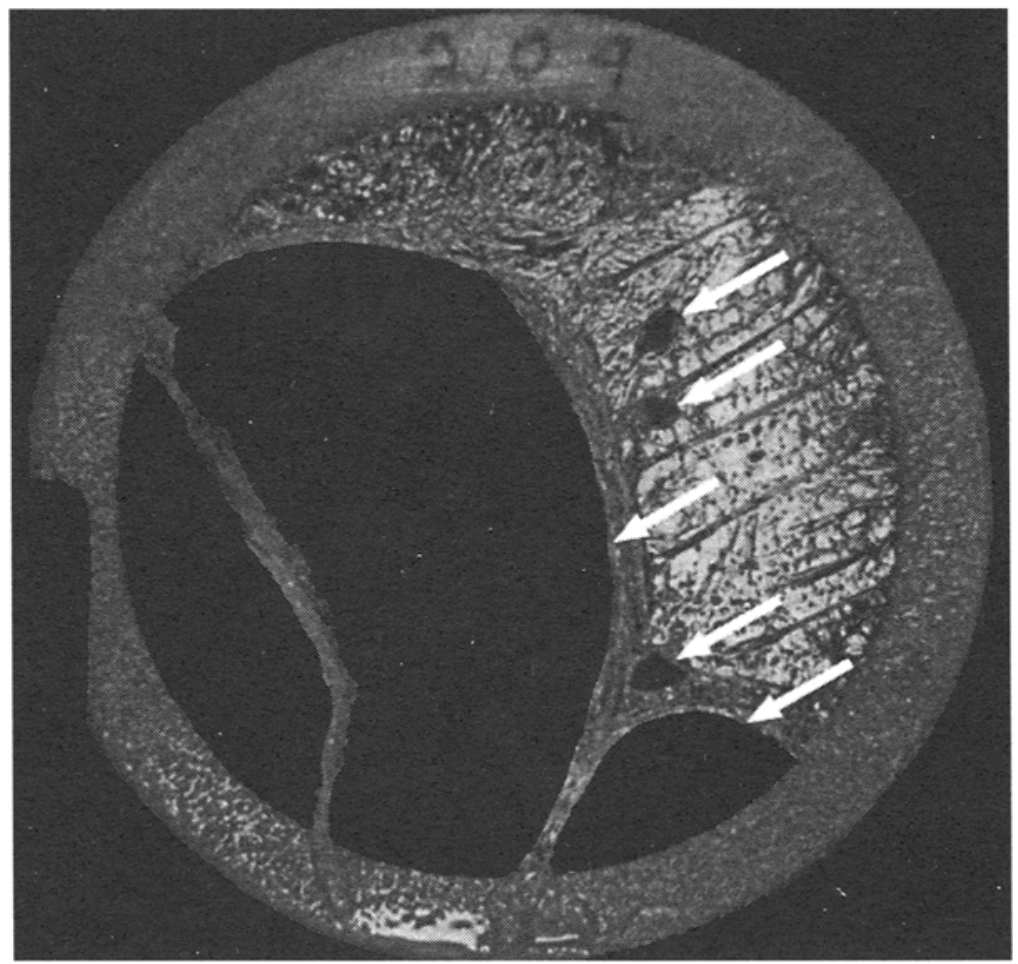

Figure 15. Damage to a membrane after 40 shock waves at $\mathrm{F} 2$ in a mixture of castor oil and glass spheres at $\alpha=160 \mu \mathrm{l}^{-1}$. The five holes that formed are indicated by arrows. Membrane is $4.1 \mathrm{~cm}$ in diameter. Membrane 209 . 
3.2b Results: Experiments with membranes at F2 in water demonstrate that collapse of cavitation bubbles in the neighbourhood of weak membrane structures has a catastrophic effect. Nitrocellulose membranes fail after only one shock wave in water by the formation of one or more holes. Large holes that are often observed could be formed by the collapse of one large bubble, or, more likely since smaller holes have also been observed, by a cloud of small bubbles. In general, damage is concentrated along the centre line where cavitation is most intense.

While membranes are not damaged in non-cavitating liquids even after more than 100 shock waves, they fail in the same liquid when it is seeded with heterogeneous scatterers. Figure 15 shows a membrane after 40 shock waves in a solution of castor oil and hollow glass spheres at a concentration $\alpha=160 \mu 1^{-1}$. We infer that the large tears on the left and bottom of figure 15 occurred earlier than the smaller ones on the right, and that the figure illustrates the progress of damage from nucleation to massive failure. The positions of the large tears suggest that with this mode of damage the failure tends to initiate at the perimeter of the sample near the wooden support or migrates in that direction after formation. The holes in this mode of failure, which we refer to as "shear" failure, tend to be more angular than with cavitation failure, though in the case of cavitation there are exceptions. Because of the variability of the present results, this characterization is preliminary and should be further refined in future research.

Figure 16 is a plot of $N_{f}$ the mean number of shocks to membrane failure vs. $\alpha$ for all liquids tested. The points lying on the vertical axis represent $N_{f}$ for the corresponding pure liquid, $\alpha=0$. In castor oil, a non-cavitating fluid, $N_{f}$ was strongly dependent on $\alpha$. Failure was observed at only one $\alpha, 160 \mu \mathrm{I}^{-1}$ (see figure 15), after $32.0 \pm 15.6$ shocks. It is expected that damage will be small at larger concentrations, when the shock is more strongly attenuated, but this hypothesis has not yet been tested. We propose that damage does not occur at lower $\alpha$ because the shock front is not sufficiently perturbed, and at higher $\alpha$ because it is too weak. If confirmed, this result would indicate that shock wave scattering in inhomogeneous material can cause membrane damage. We take this to be

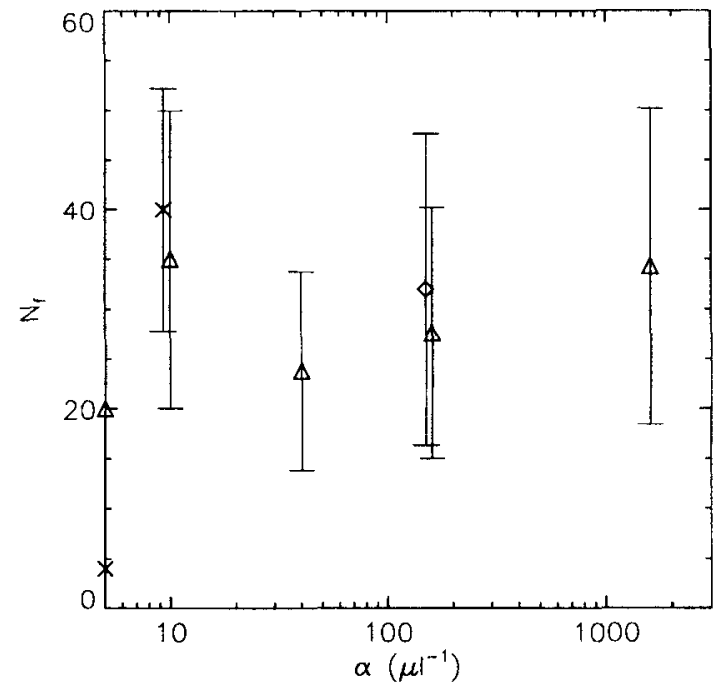

Figure 16. Number of shock waves to failure $N_{f}$ vs. concentration of spheres $\alpha . \triangle$, glycerine (57 membranes); $\diamond$, castor oil (22 membranes); $x$, ethylene glycol (four membranes). Data for two different liquids at the same concentration have been displaced horizontally for clarity. $\mathrm{Er}-$ ror bars indicate standard deviation. No damage was seen in castor oil at $\alpha=40 \mu \mathrm{I}^{-1}$. 


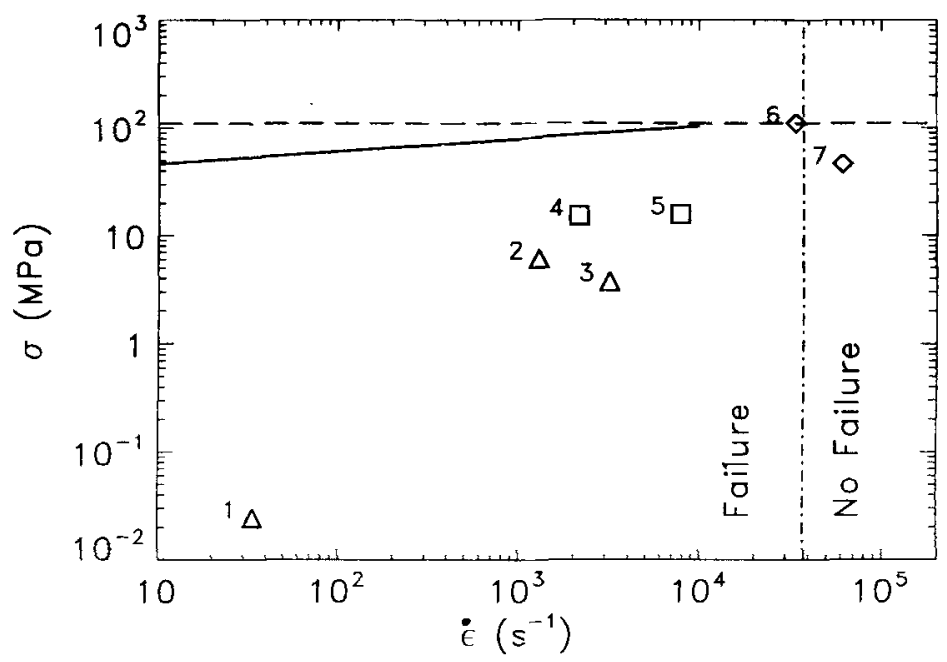

Figure 17. Failure model; stress dependence on strain rate. ---. static failure stress of nitrocellulose; - -- , boundary delimiting approximate strain rate for failure of test membranes: - _ , ultimate stress of polymer LX-04-1 (Hoge 1967). Test conditions are numbered in order of increasing strain rate.

tentative confirmation that the shear mechanism was the cause of the failure observed in seeded castor oil.

The stress calculated from the mean data from each test phantom is plotted vs. the corresponding strain rate in figure 17 . The stress correlates well with strain rate, indicating that the data are in conformity with a dynamic failure mechanism. The dashed line in figure 17 indicates the static failure stress $\sigma_{f}$. Also shown is the ultimate stress under dynamic loading of a polymeric explosive (Hoge 1967). The data of the present study are obtained under conditions in which the membranes are undergoing different levels of damage, measured by the inverse of $N_{f}$, from large at low $\dot{\epsilon}$ to negligible at high $\dot{\epsilon}$. The trend of this behaviour is steeper than that shown by the ultimate stress in dynamic failure measured by Hoge (1967). The vertical dot-dashed line in figure 17 indicates an approximate strain rate above which damage becomes negligible.

Miner (1945) showed that the fraction of loading cycles to failure that has been applied at a given stress level to a specimen is also the fraction of the useful life that has been expended. We define a dose at failure $D\left(N_{f}\right)$ by

$$
D\left(N_{f}\right)=N_{f}\left(\sigma / \sigma_{f}\right)
$$

where $\sigma$ is the average stress applied to the membrane by the focused shock waves. The resulting values of $D\left(N_{f}\right)$ are given in figure 18. The dose correlates well with strain rate. At low strain rates membranes fail with a much smaller dose than at high strain rates. To the accuracy of the present data, this analysis suggests that ESWL shock waves induce strain rates in tissue of order $10000 \mathrm{~s}^{-1}$ and that behind a tissue phantom (points numbered 4 and 5) membranes fail after a dose of about $D\left(N_{f}\right)=1400$. Above strain rates of about $40000 \mathrm{~s}^{-1}$ ESWL shocks cause virtually no damage. 


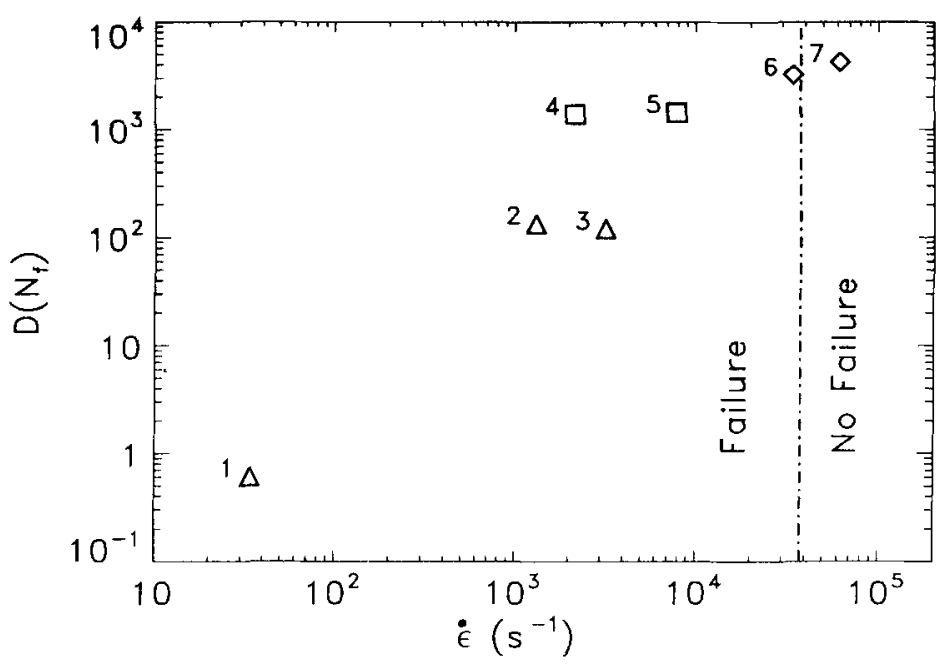

Figure 18. Dose at failure vs. strain rate. Test conditions are numbered in order of increasing strain rate.

\section{Conclusion}

Because tissue is acoustically inhomogeneous, it refracts and scatters waves. When wave fronts become curved they can focus and the wave energy can be substantially concentrated in regions far from the source. We have described two examples of the effects of focused shock waves on tissue. We suggest that wave focusing by bony tissue can concentrate wave energy generated by missile impact onto vital organs, such as the spinal cord, with consequent injury. We have explored the nature of membrane failure induced by focused shock waves in both cavitating and non-cavitating fluids. The membranes fail by a fatiguelike process during the application of many shock waves. A simple model of shearing on a small scale provides a framework for accounting for failure by dynamic fatigue, and a criterion for dose has been defined.

The author recognizes his colleagues whose work this paper reports, B Carriére, J S Kung, $S$ Wolf, J Cates, A Evan, P Blomgren and D Howard.

\section{References}

Brown-Séquard C E 1860 Course of lectures on the physiology and pathology of the central nervous system (Philadelphia: Collins)

Evan A P, McAteer J A 1996 Current perspectives on shock wave lithotripsy adverse effects. In New developments in the management of urolithiasis (eds) J Lingeman, G Preminger (New York: Igaku-Shon) pp 3-20

Evan A P, Connors B A, Willis L R, Trout A, Lingeman J E 1996a Kidney size is a determinant of structural/functional injury following shock wave treatment of pigs. J. Acoust. Soc. Am. 98: 2943 
Evan A P, Willis L R, Connors B A, Trout A, Lingeman J E 1996b Renal injury induced by clinical doses of shock waves. J. Acoust. Soc. Am. 99: 2510

Goss S A, Johnston R L, Dunn F 1978 Comprehensive compilation of empirical ultrasonic properties of mammalian tissues. J. Acoust. Soc. Am. 64: 423-457

Hoge K G 1967 The behaviour of plastic-bonded explosives under dynamic compressive loads. Appl. Polym. Symp. 5: 19-40

Howard D D, Sturtevant B 1997 In vitro study of the mechanical effects of shock wave lithotripsy. Ultrasound Med. Biol. 23: 1107-1122

Janzon B, Schantz B, Seeman T 1988 Scale effects of ballistic wounding. J. Trauma 28: 29-32

Kaude J V, Williams C M, Millnder M R, Scott K N, Finlayson B 1985 Renal morphology and function immediately after extracorporeal shock-wave lithotripsy. Am. J. Radiat. 145: 305-313

Miner M A 1945 Cumulative damage in fatigue. J. Appl. Mech. 12: A 159-A 164

Ming L, Yu-Yuan M, Rong-Xiang F. Tian-Shun F 1988 The characteristics of the pressure waves generated in the soft target by impact and its contribution to indirect bone fractures. J. Trauma 28: 104-109

Saxon M, Snyder H A, Washington J A 1982 Atypical Brown-Sequard syndrome following gunshot wound to the face. J. Oral Maxillofac. Surg. 40: 299-302

Suneson A, Hansson H A. Seeman T 1988 Central and peripheral nervous damage following high-energy missile wounds in the thigh. J. Trauma 28: 197-203

Suneson A, Hansson H A, Seeman T 1990 Pressure wave injuries to the nervous system caused by high-energy missile extremity impact: Part I. Local and distant effects on the peripheral nervous system - a light and electron microscopic study on pigs. J. Trauma 30: 281-293

Taylor R G, Gleave J R W 1957 Incomplete spinal cord injuries. J. Bone J. Surg. B39: 438-450

Williams P L, Warwick R 1989 Gray's anatomy 37th edn, (eds) M Dyson. L H Bannister (Edinburgh: Churchill Livingstone) figure 3.54 